\title{
Cytomegalovirus ileitis in an immunocompetent patient
}

\author{
María Auxiliadora Tejedor-Cerdeña ${ }^{1}$, Antonio Velasco-Guardado ${ }^{1}$, Ana Fernández-Prodomingo ${ }^{1}$, \\ María Concepción Piñero-Pérez ${ }^{1}$, Renzo Calderón ${ }^{1}$, Ana Beatriz Prieto-Bermejo ${ }^{1}$, \\ Ana Sánchez-Garrido ${ }^{1}$, Juan Martínez-Moreno ${ }^{1}$, Fernando Geijo-Martínez ${ }^{1}$, Óscar Javier Blanco-Múñez ${ }^{2}$ \\ and Antonio Rodríguez-Pérez ${ }^{1}$
}

Departments of ${ }^{1}$ Digestive Diseases, and ${ }^{2}$ Pathology. Hospital Universitario de Salamanca. Spain

\begin{abstract}
Cytomegalovirus (CMV) is a virus that belongs to the family of Herpesviridae. Infection can cause a serious disease in immunocompromised patients, but it can also affect immunocompetent patients, creating generally self limiting symptoms. However, in some cases it can be fatal. We present a case of CMV ileitis with serious clinical symptoms that led to an operation in an immunocompetent patient.
\end{abstract}

Key words: Ileitis. Cytomegalovirus. Immunocompetent.

Tejedor-Cerdeña María Auxiliadora, Velasco-Guardado Antonio, Fernández-Prodomingo Ana, Piñero-Pérez María Concepción, Calderón Renzo, Prieto-Bermejo Ana Beatriz, Sánchez-Garrido Ana, Martínez-Moreno Juan, Geijo-Martínez Fernando, BlancoMúñez Óscar Javier, Rodríguez-Pérez Antonio. Cytomegalovirus ileitis in an immunocompetent patient. Rev Esp Enferm Dig 2011; 103: 154-156.

\section{INTRODUCTION}

Cytomegalovirus (CMV) is a form of Herpes virus. In humans, it is known as human herpesvirus 5 (HHV-5). It belongs to the Betaherpesvirinae subfamily of Herpesviridae. The source of the virus name is the size in-

Received: 01-06-10.

Accepted: 16-06-10.

Correspondence: María Auxiliadora Tejedor Cerdeña. Servicio de Aparato Digestivo. Hospital Universitario de Salamanca. Po San Vicente, n. ${ }^{\circ}$ 58182. 37007 Salamanca. Spain

e-mail: maria_tc_4@hotmail.com crease observed in the infected cells as a result of the weakening of the cytoskeleton. It can cause serious diseases in immunocompromised patients, either due to the reactivation of a latent infection or to a primo-infection. The CMV infection mainly affects immunocompromised patients. However, it is not rare in immunocompetent patients, and its symptoms are compatible with a non-specific viral syndrome or they simulate a mononucleosis $(1,2)$.

CMV infection can affect any system of the body, which, in decreasing order of incidence are: gastrointestinal tract (mainly in the form of colitis), central nervous system (meningitis, encephalitis,...), hematological manifestations (hemolytic anemia, thrombocytopenia), eyes (retinitis, uveitis), liver (hepatitis), lung (pneumonitis) and circulatory system (venous and arterial thrombosis) (3).

In the case of gastrointestinal tract, the most common affected areas are the colon and the rectum, and other locations such as the small intestine are rarer. Clinical symptoms include: fever, abdominal pain, anorexia, nausea, vomiting, diffuse abdominal pain, lower abdominal pain, weight loss, diarrhea, hematochezia or melena. Complementary tests for diagnosis include: abdominal ultrasound or CT scan, gastroscopy and colonoscopy with ileoscopy with biopsies and fecal culture. Endoscopic findings include friable or edematous mucosa, fold thickening and, more characteristically, well-delimited medium- or bigsized ulcers with protruding edges separated by normal mucosa. The diagnosis is based on histological analysis, fecal culture, C-reactive protein and serology $(4,5)$. The pathogenesis of these lesions is still unclear, but it seems to be the consequence of ischemic damage to the mucosa secondary to an infection of the vascular endothelial cells and local autoimmune alterations (6).

Although this infection is considered to be self-limiting in immunocompetent patients, some cases with serious clinical manifestations that can lead to death have been published (3). 


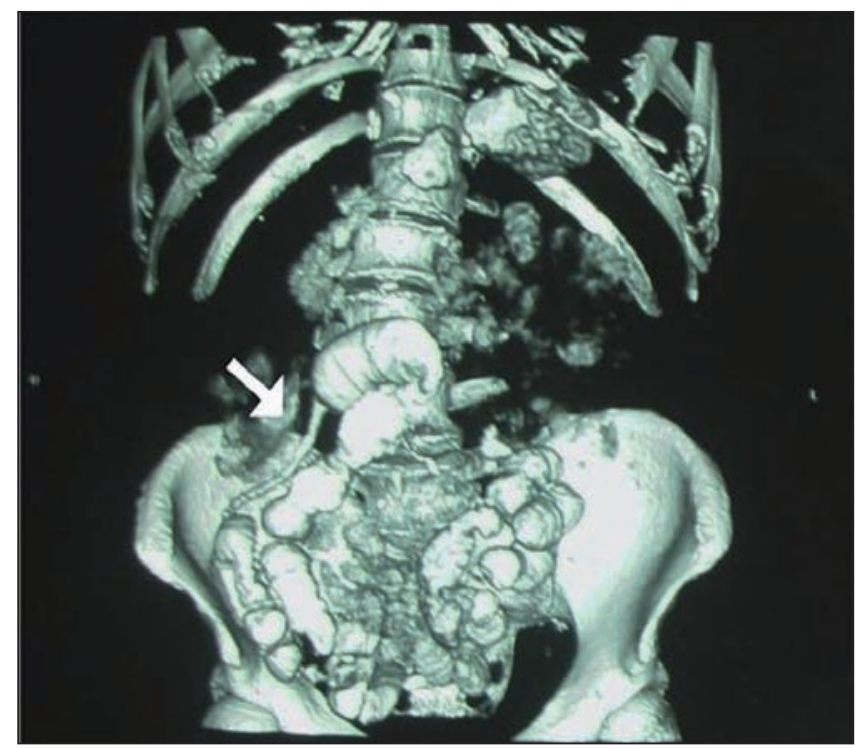

Fig. 1. Three-dimensional reconstruction of the CT scan that shows the 18-cm ileum stenosis (arrow).

\section{CLINICAL CASE}

85-year old male patient with a personal record of arterial hypertension, dyslipidemia, acute myocardial infarction with a residual ventricular aneurysm, transient ischemic cerebrovascular attacks, benign prostatic hyperplasia and renal cysts with normal renal functions. No toxic habits were registered. The patient is under treatment with metoprolol, cardioaspirin and tamsulosin. The patient did not undergo prior operations.

The patient was admitted in the high-resolution department of the digestive diseases with symptoms of 15 days of evolution that include episodes of colicky, non-referred periumbilical abdominal pain, intermittent alimentary vomiting and poor appetite. He did not present fever or alterations of the intestinal rhythm. The first analysis revealed: glucose: $119 \mathrm{mg} / \mathrm{dl}$; urea: $22 \mathrm{mg} / \mathrm{dl}$, creatinine: $1.14 \mathrm{mg} / \mathrm{dl}$; Na: $142 \mathrm{mmol} / \mathrm{k}$; K: 4.3 mmol/l; Hb: $13.2 \mathrm{~g} / \mathrm{dl}$; hematocrit: 41.2\%; mean corpuscular volume: $93.8 \mathrm{fl}$; leukocytes: $13.42 \times 10^{3} / \mu \mathrm{l}$; neutrophiles: $85.7 \%$; platelets: $149 \times 10^{3} / \mu \mathrm{l}$; prothrombin time: $73 \%$. The gastroscopy showed a hiatus hernia with preserved mucosa, as well as areas of intestinal metaplasia in the stomach with surface erosions. The urease test was negative and the abdominal ultrasound did not present significant findings. Afterwards, and in view of the increase in the symptoms, which were accompanied by slight fever, the patient was hospitalized. A colonoscopy was performed, advancing up to 3-4 cm of the terminal ileum, but further progression was not possible due to stenosis. A CT scan (Fig. 1) revealed a $18-\mathrm{cm}$ stenosis of the ileum.

After the CT scan, the patient presented signs of peritoneal irritation and distension in the abdominal examination. Therefore, after an assessment from the department

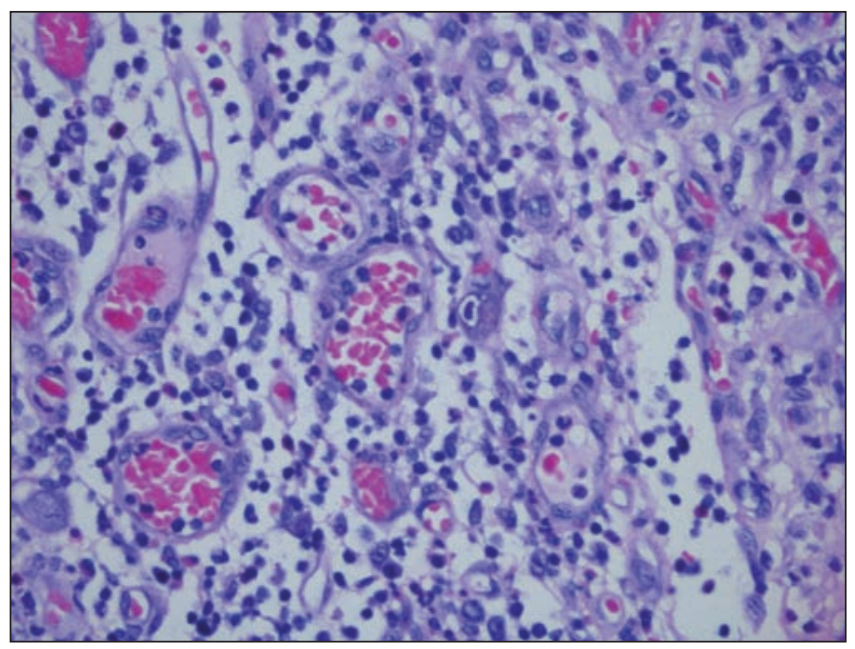

Fig. 2. Large stromal cells with granular cytoplasma and intranuclear inclusions surrounded by a white halo (H\&E, 400x).

of surgery, we decided to perform an emergency operation. The ileum and the appendix were resected. The resected piece revealed a stenosed segment with firm, thickened walls, which measured $8 \mathrm{~cm}$ longitudinally and which was compatible, after it was opened, with a region of wide mucous ulceration with several pseudopolypoid formations inside.

The anatomopathological analysis showed areas of preserved mucosa with marked inflammatory changes, mainly cryptal distortion, with a slight loss of epithelial mucous secretion, superficial lymphangiectasia and lymphoplasmacytic infiltrate of the lamina propria, together with several eosinophils and neutrophils, which spread transmurally, mainly in ulcerated areas.

The ulcerated foci, showed a healthy and partially reepithelialzed granulation tissue, present some big cells, mainly fibroblasts and endothelial cells, with granular cytoplasm and a big nucleus, with eosinophilic inclusions surrounded by a white strip inside (Fig. 2). Further immunohistochemical techniques proved the presence of cytomegalovirus infection in those cells (Fig. 3).

After surgery, the patient's condition improved without antiviral therapy, and he was discharged. He remains asymptomatic and follows no specific treatment.

\section{DISCUSSION}

CMV infection in immunocompetent patients is a rare pathology, and there are few documented cases of a segmented involvement of the ileum $(4,7)$. There are several cases of enteritis associated to colitis (8) or of colonic involvement on its own (9) in the literature. An important factor in all these cases is the age of the patients, which is usually over 55 years. The higher incidence of this pathology in older immunocompetent patients might be 


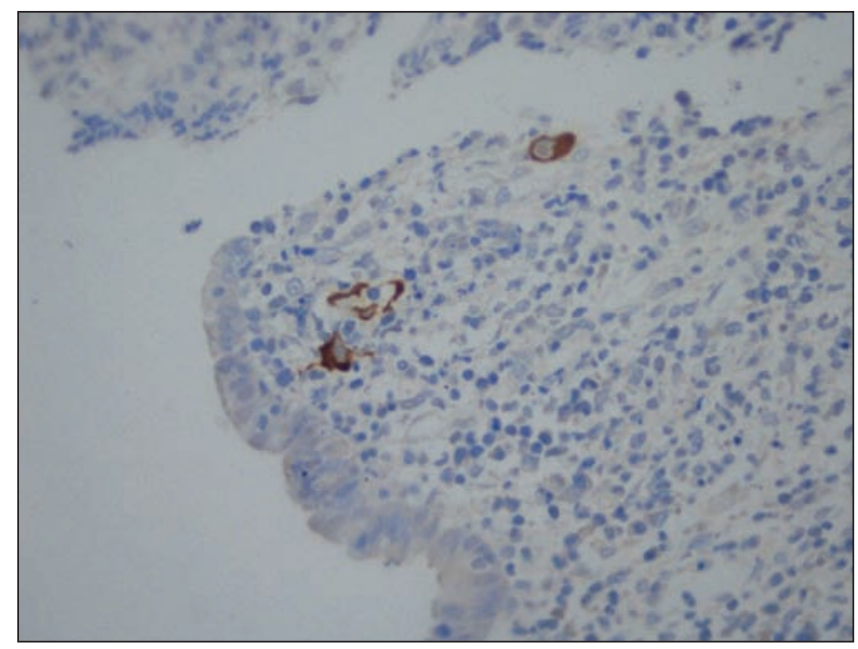

Fig. 3. Immunohistochemical techniques with cytomegalovirus infection $(400 \mathrm{x})$.

related to a dysfunction in B- and T-lymphocytes as time passes, as well as to a dysfunction of cytokines and the immunological integrity of the mucosa. All these factors could create a relative immunodeficiency that predisposes the patients to inflammatory or infectious diseases (10). A meta-analysis with 43 cases of CMV colitis in immunocompetent patients, most of them was more than 55 years old and they presented comorbidities (77.3\%). In this group, $47 \%$ of them presented conditions that induced alterations of the immune system, such as pregnancy, renal failure, diabetes mellitus or untreated cancer (non-hematologic) (9). Another review with 44 patients with CMV colitis showed an average age of 63 years (11).

The treatment of choice of CMV infection are antiviral drugs, such as ganciclovir or foscarnet. In a meta-analysis by Galiatsatos in 2005 (9), patients who were less than 55 years old and had no other concomitant disease showed a spontaneous remission of the infection. These results suggest that in some specific immunocompetent patients, antiviral therapy might not be necessary. These conclusions are also shared by other authors, who consider antiviral drugs to be unnecessary in immunocompetent patients (8). However, more randomized studies are necessary in order to confirm these observations.

The mortality of this infection ranges between 6.2 and $32 \%$, and the higher values can be seen in patients who are more than 55 years old and present diseases that affect their immune response. Age, male sex, presence of comorbidities, need for surgery and nosocomial infections are related to a lower survival (3).

\section{REFERENCES}

1. Sissions JPG, Carmichael AJ. Clinical aspects and management of cytomegalocirus infection. J Infect 2002;44(2):78-83.

2. Cheung $\mathrm{AN}, \mathrm{Ng}$ IO. Cytomegalovirus infection of the gastrointestinal tract in non-AIDS patients. Am J Gastroenterol 1993;88(11): 1882-6.

3. Rafailidis PI, Mourtzoikou EG, Varbobitis I, Falagas ME. Severe cytomegalovirus infection in apparently immunocompetent patients: a systematic review. Virol J 2008;5:47.

4. Taniwaki S, Kataoka M, Tanaka H, Mizuno Y, Hirose M. Multiple ulcers of the ileum due to cytomegalovirus infection in a patient who showed no evidence of an immunocompromised state. J Gastroenterol 1997;32:548-52.

5. Keates J, Lagahee S, Crilley P, Haber M, Kowalski T. CMV enteritis causing segmental ischemia and massive intestinal hemorrhage. Gastrointest Endosc 2001;53:355-9.

6. Goodgame RW. Gastrointestinal cytomegalovirus disease. Ann Intern Med 2003;119:924-35.

7. Ryu KH, Yi SY. Cytomegalovirus ileitis in an immucompetent elderly adult. Word J Gastroenterol 2006;12(31):5084-6.

8. Sakamoto I, Shirai T, Kamide T, Igarashi M, Koike J, Ito A, et al. Cytomegalovirus enterocolitis in an immunocompetent individual. Clin Gastroenterol 2002;34:243-6.

9. Galiatsatos P, Shrier I, Lamoureux E, Szilagyi A. Meta-analysis of outcome of cytomegalovirus colitis in immunocompetent host. Dig Dis Sci 2005;50:609-16.

10. Schmucker DL, Heyworth MF, Owen RL, Daniels CK. Impact of aging on gastrointestinal mucosal immunity. Dig Dis Sci 1997;42 (3):694-5.

11. Karakozis S, Gongora E, Caceres M, Brun E, Cook JW. Life-threatening cytomegalovirus colitis in the immunocompetent patient: report of a case and review of the literature. Dis Colon Rectum 2001;44 (11):1716-20. 